BMJ Open Gastroenterology

\title{
Non-diarrhoeal increased frequency of bowel movements (IFoBM-ND): enterovirus association with the symptoms in children
}

\author{
C Durga Rao, ${ }^{1}$ P P Maiya, ${ }^{1,2}$ M Ananda Babu ${ }^{1,3}$
}

To cite: Rao CD, Maiya PP, Babu MA. Non-diarrhoeal increased frequency of bowel movements (IFoBM-ND): enterovirus association with the symptoms in children. BMJ Open Gastro 2014;1: e000011. doi:10.1136/ bmjgast-2014-000011

Received 21 June 2014 Revised 30 July 2014 Accepted 3 August 2014
${ }^{1}$ Department of Microbiology \& Cell Biology, Indian Institute of Science, Bangalore, Karnataka, India ${ }^{2}$ Department of Paediatrics, M. S. R. T. Hospital, and Agadi Hospital, Bangalore, Karnataka, India

${ }^{3}$ Department of Paediatrics, R. M. V. Hospital, and Arpita Clinic, Bangalore, Karnataka, India

Correspondence to Professor Durga C Rao; cdr@mcbl.iisc.ernet.in

\section{ABSTRACT}

Objective: Infectious and non-infectious causes are associated with increased frequency of bowel movements (IFoBM). But, a viral aetiology to nondiarrhoeal IFoBM (IFoBM-ND) has not been described. Owing to an accidental infection by an echovirus 19 strain, persistent diarrhoea-associated virus, isolated from a child with persistent diarrhoea, DCR experienced persistent IFoBM-ND with an urgency to pass apparently normal stools more than once each day for about 3 months. A follow-up study was undertaken to determine the prevalence of IFOBM-ND, and association of non-polio enteroviruses (NPEVs) with the symptom in infants from birth to 2 years.

Design: A cohort of 140 newborns was followed for 6 months to 2 years from birth for IFoBM-ND. Stool samples collected every 14 days were examined for NPEVs, rotavirus and other viral/bacterial agents for their possible association with IFoBM-ND and diarrhoea.

Results: Of 403 NPEV infection episodes among 4545 oral polio vaccine strains-negative stool samples, approximately $29 \%$ were associated with IFoBM-ND ( $15 \%$ acute and $14 \%$ persistent), including resolution of $74 \%$ of constipation episodes, and $18 \%$ with diarrhoea, suggesting that about $47 \%$ of NPEV infection episodes in children below 2 years of age are associated with gastrointestinal symptoms. About $83 \%$ of IFoBM-ND episodes are associated with the NPEV infection and $17 \%$ of the episodes are of unknown aetiology.

Conclusions: NPEV is the single most frequently detected viral agent in children with IFOBM-ND and its association with the symptom is highly significant, warranting detailed investigations on the role of NPEVs in gastrointestinal diseases.

\section{BACKGROUND}

The frequency of bowel movements (BM) refers to the number of times a person discharges stool from the large intestine each day. There is no general rule for normal frequency of BM, which varies from three times a day to three times a week, and from person

\section{Summary box}

What is already known about this subject?

- Bowel movements (BM) is perhaps the least understood and less studied of all human bodily functions.

- BM with less than three movements a week is considered as constipation, and more than three watery stools a day as diarrhoea.

- However, non-diarrhoeal increased frequency of BM (IFoBM-ND) in children has not been considered as a clinical symptom associated with viral infections.

What are the new findings?

- This follow-up study of a cohort from birth up to 2 years of age reveals for the first time that non-polio enterovirus (NPEV) infections are associated with acute and persistent IFoBM-ND in children.

- NPEV-IFOBM-ND accounted for about $29 \%$ of NPEV infection episodes and $83 \%$ of total IFoBM-ND episodes.

- NPEV infections appear to result in resolution of about $74 \%$ of constipation episodes.

How might it impact on clinical practice in the foreseeable future?

- The present finding of NPEV association with IFoBM in children, including acute and persistent IFoBM-ND and diarrhoea, could impact on future clinical understanding of IFoBM-ND and its recognition as a gastrointestinal disease symptom in clinical practice, and promote active research towards antienteroviral therapeutics.

to person. However, it is commonly considered that a great majority of people have one $\mathrm{BM}$ each day. ${ }^{1-5} \mathrm{~A}$ variety of conditions may cause a significant increase in the frequency of BM, which include bacterial, parasitic or viral infections of the gastrointestinal tract, coeliac disease, cancer of the digestive tract, food allergies, diverticulitis, gall bladder disease or stones, lactose intolerance, inflammatory bowel disease (Crohn's disease and 
ulcerative colitis), irritable bowel syndrome (IBS), certain medications or drug overdose, etc. ${ }^{6-11}$ Increased frequency of $\mathrm{BM}$ can be either diarrhoeal, referred to as IFoBM-D, or non-diarrhoeal (IFoBM-ND). Frequent BM can be associated with a serious disease such as bowel obstruction and pancreatitis leading to a life-threatening condition. Other digestive as well as non-digestive symptoms including abdominal pain or cramping, bloody stool, diarrhoea, faecal incontinence, gas, abdominal swelling or bloating, nausea with or without vomiting, painful BM, urgent need to pass stool, body aches and fever might occur along with frequent BM. ${ }^{2}$

The majority of studies on the frequency of BM are based either on data recorded in sets of children of different age groups, ${ }^{512-14}$ or on the recording of BM only at specific months after birth ${ }^{15}$ or information provided by parents of children of specific age to a bowel-habit questionnaire. ${ }^{16}{ }^{17}$ Further, significant differences in BM frequency appear to exist between children in developed and developing countries due to ethnic differences in dietary factors. ${ }^{4}$ Most of these studies suffer from severe variation in design and validity, and are only moderately sensitive. ${ }^{18}$ To the best of our knowledge, there is no systematic follow-up study on a day-to-day basis on frequency and type of BM, both IFoBM-D and IFoBM-ND, and analysis of the causes for change in BM frequency in a cohort of children from birth to 2 years of age.

The genus enterovirus (EV), belonging to the family Picornaviridae, consists of a large group of viruses ${ }^{19} 20$ that are associated with a wide variety of diseases in humans including acute flaccid paralysis, aseptic meningitis, encephalitis, type-1 diabetes, hand-foot-and-mouth disease, uveitis, cardiomyopathy, aplastic anaemia, coagulopathy, multisystem haemorrhagic disease, sudden deafness, fatal illness with pulmonary hypertension in neonates, diarrhoea, etc. ${ }^{19-29}$ Poliovirus, coxsackievirus (CVA and CVB), echovirus (E), newer EVs and rhinovirus represent major pathogenic species of humans in the EV genus, and together present about 200 serotypes. ${ }^{19} 20$

A recent long-term epidemiological study in Bangalore, India, from our laboratory revealed a significant association of non-polio EVs (NPEVs) with acute diarrhoea in children. ${ }^{23}$ Further, a follow-up of 140 infants from birth for 6 months to 2 years of age revealed significant association of NPEV infections with acute diarrhoea as well as persistent diarrhoea (PD).$^{30}$

During the large-scale purification of an echovirus 19 (E19) strain isolated from a child suffering from PD, called the persistent diarrhoea-associated virus (PDV; GenBank Accession number JX513514) for antibody generation, DCR had accidental infection resulting in a symptom, referred to as "non-diarrhoeal persistent IFoBM' (PIFoBM-ND), which lasted for about 3 months. This symptom is often characterised by intense BM, an urgency to pass apparently normal stools more than twice a day instead of the normal once a day, abdominal bloating and discomfiture. This infection also resulted in a mild loss of motor function of the right hand, leading to stammering and incoherent handwriting. PDV was detected in the stools for more than 2 months, followed by E6 strain for about 12 days. The symptom subsided after a final mild diarrhoeal episode involving an enteropathogenic Escherichia coli infection for 3 days. Although IFoBM-ND shares some symptoms associated with IBS $^{2}{ }^{31-33}$ such as urgency for BM, discomfort, a feeling of incomplete evacuation (tenesmus) and abdominal bloating, as was experienced by $\mathrm{DCR}^{30}$ comorbidities such as chronic abdominal pain, diarrhoea or constipation, headache, backache, fibromyalgia, chronic fatigue syndrome or psychiatric symptoms such as depression and anxiety frequently associated with IBS $^{31-33}$ were not observed.

This unusual clinical symptom prompted us to undertake the present follow-up study of a cohort of infants from birth up to 2 years of age to understand the frequency of NPEV infections and their associated clinical outcomes including diarrhoea and IFoBM-ND. In a recent report, we described the frequency of NPEV infections, and association of $18 \%$ of the NPEV infection episodes with diarrhoea (12\% acute and $6 \%$ persistent) in this cohort. ${ }^{23}$ We present an analysis of the apparently asymptomatic (referred to as non-diarrhoeal in our recent report ${ }^{23}$ ) NPEV infection episodes and their association with IFoBM-ND as observed in the cohort in the community setting.

\section{METHODS \\ Definitions}

BM or bowel habit is referred to as discharge or passing of stool from the large intestine, or defaecation. Frequency of $\mathrm{BM}$ is the number of times an individual passes stools each day. BM ranges from three times a day to three times a week among different individuals. Less than three movements a week is considered as constipation $^{34}$ and more than three watery stools a day is considered as diarrhoea. ${ }^{1}{ }^{2}$ In this study, passing more than three apparently normal stools in a day or twice that of the existing frequency is considered as IFoBM-ND, the majority of acute IFoBM-ND (AIFoBM-ND) episodes lasting for less than 7 days and a few extending up to 14 days, and PIFoBM-ND episodes lasting for more than 14 days and up to three or more months. The corresponding IFoBM episodes involving watery stools are similarly considered as acute diarrhoea (AIFoBM-D) and PD (PIFoBM-D). ${ }^{35} 36$

\section{The cohort}

The cohort, consisting of 140 infants, was recruited from birth over a period of 24 months between 2009 and 2010. All infants were normal and healthy at birth and remained normal during the study period. The major criteria for selection are the willingness of the parents to participate in the long-term study, and that they do not move out of the city during the study period. The 
mother was trained to carefully monitor and record the number and type of stools (diarrhoeal and nondiarrhoeal) daily, and to collect a stool sample in a sterile container on the designated date. The children were located within a radius of $6 \mathrm{~km}$ and the mothers were accessible by phone. R. M. V. Hospital, M. S. Ramaiah Teaching Hospital, Agadi Hospital and Arpita Clinic served as nodal centres for the 2-year follow-up study. More details on the cohort are described recently. ${ }^{23} 30$

\section{Stool samples}

Stool samples were collected once in 14 days, and on alternate days during a diarrhoeal episode or an infection episode identified by NPEV or rotavirus (RV) positivity. ${ }^{23} 30$ The samples were transported from the residences of children to the laboratory on ice the same day.

\section{Oral polio vaccine period}

Almost every child in India receives oral polio vaccine $(\mathrm{OPV})$ six to seven times during the first 2 years of life. Although a majority of OPV recipients shed vaccine strains for less than 7 days, a significant number of children were found to shed the virus up to 14 days and a few beyond 14 days. ${ }^{22} 23{ }^{30}$ Hence, the clinical protocol should distinguish samples positive for OPV strains to reduce the burden of sequencing them from the large number of samples, which become EV positive in cell culture and reverse transcription PCR (RT-PCR). The 14-day period following OPV administration is referred to as the OPV period, and the majority of the samples positive for virus growth in rhabdomyosarcoma or HeLa cells, except the 100 random samples collected during this period, are considered OPV positive and are not subjected to RT-PCR and sequence analysis. However, all strains positive in the diarrhoea samples were identified by sequencing the VP1 gene. ${ }^{22} 2330$

\section{Identification of EV, RV and other agents in stool samples}

Methods for detection of NPEVs and other picornaviruses (kobuvirus, classevirus and cardiovirus), RV, diarrhoeagenic E. coli were described previously. ${ }^{22} 2330$ The serotypes of NPEVs were identified by comparison of complete VP1 gene sequences with the prototype and other NPEV sequences in the GenBank database using the BLAST programme and by phylogenetic analysis. ${ }^{22} 233037$ Nucleotide sequencing was carried out at Macrogen (Korea).

\section{Ethics}

Approvals from the Institutional Biosafety Committee and Ethical Committee were obtained for carrying out the work.

\section{Statistical analysis}

The data in Microsoft Office Excel format were subjected to statistical analyses using the Fisher exact test, the student $t$-test and computed $p$ values as described..$^{30} 38$

\section{RESULTS}

\section{Age-dependent frequency of BM}

Owing to unexpected dropouts, the follow-up period for different children varied from 6 months to 2 years. ${ }^{30}$ For convenience, the study duration was divided into four periods of 6 months each. Analysis of the frequency of passing of stools and type of stools (diarrhoeal and normal) in the cohort recorded daily during the follow-up period revealed that the frequency of BM ranged between 0 and 16 during the first 6 months of life, between 0 and 12 during the 7-12-month period, between 0 and 8 for the 13-18-month period and between 0 and 4 during the 19-24-month period. The mean frequency among the cohort children for the four successive 6-month periods was 3.3, 1.6, 1.2 and 0.98, respectively (figure 1 ). Although the daily frequency of $\mathrm{BM}$ in a majority of the children ranged between 1 and 3 , some infants passed stools once in 2-4 days or 5-10 days on more than one occasion (figure 2). Very few infants had $1 \mathrm{BM} /$ day $(<4 \%$ of total $\mathrm{BM})$ during the first 6-month period, but the number of children having 1 $\mathrm{BM} /$ day increased with age with $>92 \%$ having passing 1 $\mathrm{BM} /$ day by 2 years of age. The frequency of BM is high in all children below 6 months of age with 2, 3-4 and 5-16 $\mathrm{BM} /$ day representing $15 \%, 23 \%$ and $34 \%$, respectively. Of note, while about $7 \%$ and $17 \%$ of the non-diarrhoeal BM occurred once in 2-4 days and 5-10 days, respectively, in children below 6 months of age, this category of BM represented less than $1-2 \%$ in children older than 6 months. The frequency of BM ranged between 0 and $16 /$ day among the children in all age groups. Approximately $87 \%$ of IFoBM-ND episodes occurred during the first year with $>57 \%$ occurring during the first 6 months. While about $82 \%$ and $10 \%$ of children in the age group of 12-18 months had 1 and 1-2 BM/day, respectively, about $97 \%$ of children in the age group of

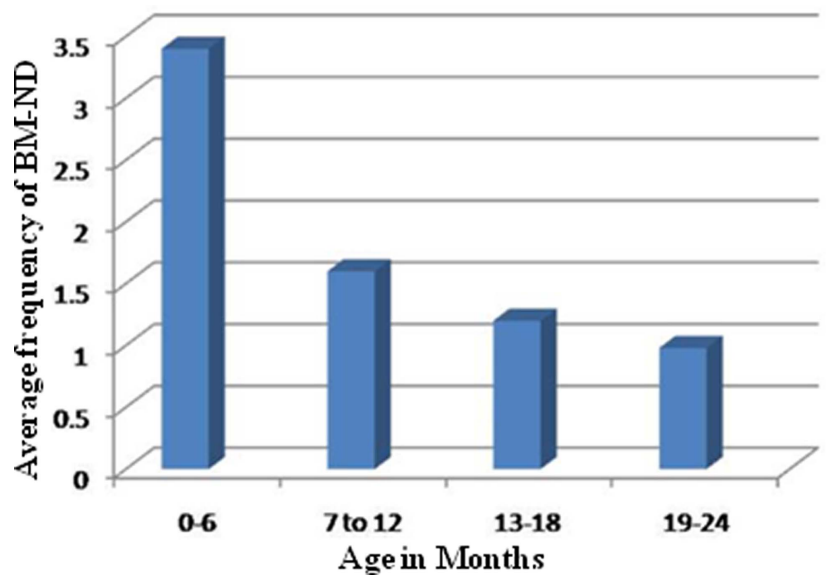

Figure 1 Age-dependent average frequency of non-diarrhoeal bowel movements (BM-ND) in the cohort. The 2-year follow-up duration is divided for convenience into four 6 -month periods. The frequency of non-diarrhoeal BM per day during the four successive 6-month periods from birth ranged between 0-16, 0-12, 0-8 and 0-4, respectively. 


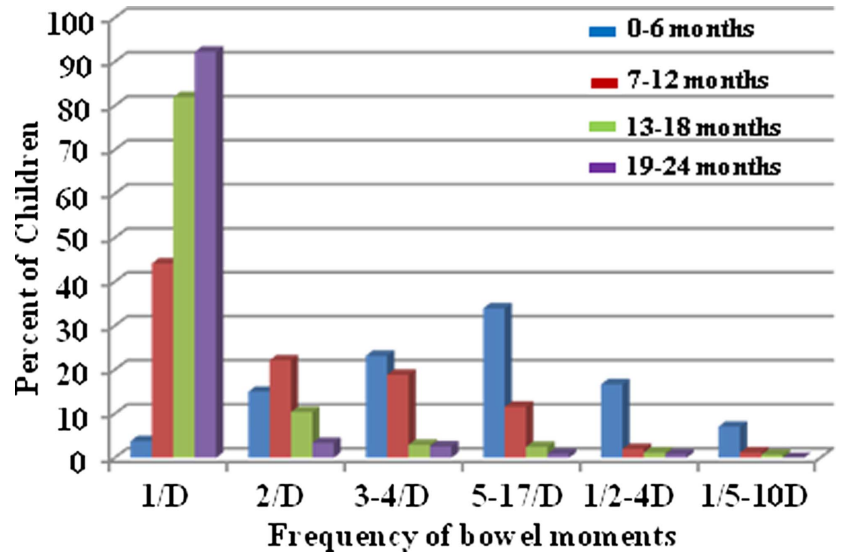

Figure 2 Age-dependent changes in bowel movement (BM) frequencies. BM frequencies were grouped into six categories, 1 in a day (1/D), 2 in a day (2/D), 3-4 in a day (3-4/D), 5-17 in a day, 1 in $2-4$ days (1/2-4D) and 1 in 5-10 days (1/5-10D). The per cent proportion of non-diarrhoeal BM of each category in each age group is calculated with reference to the total number of non-diarrhoeal BM of the respective category occurring in all the four age groups.

19-24 months had only $1 \mathrm{BM} /$ day. It should be noted that as a tradition, almost every infant in India is trained to pass stools every day in the morning by the time the child reaches $1-2$ years of age.

\section{EV association with IFoBM-ND}

Since DCR experienced PIFoBM-ND for about 3 months following accidental infection with the E19 strain, PDV, isolated from a child with PD, the cohort children were carefully monitored each day for the frequency of passing of stools. The stool samples were examined for the presence of NPEVs, and the data were analysed for correlation between changes in frequency of $\mathrm{BM}$ and NPEV infection. Of 403 NPEV infection episodes observed among the 140 children during the follow-up period, ${ }^{23}{ }^{30} 115$ were IFoBM-ND episodes. Further, 24 IFoBM-ND episodes that were negative for NPEV infection were also observed. Thus while NPEV-positive and NPEV-negative IFoBM-ND episodes represented 83\% $(115 / 139)$ and $17 \%(24 / 139)$ of total IFoBM episodes, respectively, the former accounted for $28.5 \%$ (115/403) of total NPEV infection episodes (table 1). The NPEV-negative IFoBM-ND episodes were all acute and represented about $17 \%$ of the episodes (table 1).

While acute NPEV infections ranged between $64 \%$ and $72 \%$, persistent infections were between $28 \%$ and $36 \%$ in all the age groups. Among 115 NPEV-positive IFoBM-ND episodes, acute and persistent episodes occurred at similar frequencies $(61$ acute $(53 \%)$ and 54 persistent $(47 \%)$ ) with greater than $70 \%$ of both types of episodes occurring during the first year of life. AIFoBM-ND episodes accounted for about $15 \%$ of the total NPEV infection episodes and PIFoBM-ND episodes accounted for $>13 \%$ (figure 3 and table 1 ).
NPEV serotypes associated with IFoBM-ND

We recently identified 24 NPEV serotypes associated with acute diarrhoea and 16 types with PD in this cohort. ${ }^{30}$ In the present analysis, of the 20 NPEV types detected, only 5 types E1, E6, E11, E19 and E24 were more frequently associated with IFoBM-ND. E6, E11 and E19 were frequently observed to persist for extended periods in PIFoBM-ND episodes, involving monotypic and sequential infections by different serotype strains (table 2). Note that many of the PIFoBM-ND episodes are repeated against different serotypes in table 2 due to sequential infections involving strains belonging to several NPEV serotypes during many of the episodes.

\section{Comparative analysis of diarrhoeal and IFoBM-ND episodes}

Analysis of IFoBM-ND and diarrhoeal episodes revealed that while diarrhoeal episodes accounted for $18 \%,{ }^{30}$ IFoBM-ND accounted for about 29\% (115/403) of the NPEV infection episodes. Thus, a significantly greater number of NPEV infections is associated with IFoBM-ND than with diarrhoea. While NPEV-associated diarrhoeal episodes accounted for about 38\% (73/193), and RV-associated diarrhoea for $22 \%$ (42/193), those due to other agents represented about $34 \%(66 / 193)$ of the total diarrhoeal episodes (table 1) ${ }^{30}$ Taking into consideration our recent observation that about $18-21 \%$ of diarrhoeal cases reporting to paediatricians are NPEV positive, ${ }^{23}$ about half of the NPEV-associated diarrhoeal episodes occurring in the community appear to remain unreported in India. ${ }^{23} 30$

In our recent analysis of diarrhoeal episodes in the cohort, prior to analysis of IFoBM-ND symptoms, all NPEV infection episodes of IFoBM not associated with diarrhoea were considered as non-diarrhoeal or asymptomatic. ${ }^{30}$ Analysis revealed that about $35 \%$ (115/330) of the apparently asymptomatic NPEV infection episodes and $29 \%(115 / 403)$ of the total NPEV infection episodes were associated with IFoBM-ND symptoms. Thus, NPEV symptomatic infections (diarrhoea $(18-21 \%)^{23} 30$ and IFoBM-ND (29\%)), excluding NPEV-RV-positive mixed diarrhoeal infections, accounted for about $50 \%$ of total NPEV-positive infection episodes.

Of interest, among the three highly prevalent NPEV serotypes (EV1, EV6 and EV11) associated with IFoBM-ND, E6 and E11 types were also frequently detected in diarrhoeal samples, but E1 is rarely associated with diarrhoea. Many serotypes were associated with symptomatic infections (diarrhoea and IFoBM-ND) and asymptomatic infections. While EV serotypes E21, E25 and EV83 were detected only in IFoBM-ND and asymptomatic infections, E5, EV75 and CVB5 were detected only in diarrhoeal samples ${ }^{30}$ (table 2).

Similar to that observed in PD episodes, ${ }^{30}$ PIFoBM-ND episodes were characterised either by persistence of a single strain for a prolonged period or sequential infection by multiple strains during the persistent episode (table 2). While all AIFoBM-ND episodes involved 
Table 1 Analysis of IFoBM-ND episodes in comparison to diarrhoeal episodes

\begin{tabular}{|c|c|c|c|c|c|c|}
\hline & Subjects, samples, episodes & 0-6 months & 6-12 months & 12-18 months & 18-24 months & Total (\%) \\
\hline 1 & Cohort children followed $(n=140)$ & 140 & 130 & 112 & 70 & 140 \\
\hline 2 & Total samples & 1724 & 1603 & 1381 & 864 & 5572 \\
\hline 3 & Total OPV-negative samples & 1078 & 1430 & 1268 & 769 & 4545 \\
\hline 4 & Total NPEV-positive samples & 260 & 179 & 135 & 84 & $638 / 4545(14.0)$ \\
\hline 5 & Total NPEV-negative samples & 818 & 1251 & 1133 & 685 & $3907 / 4545(86.0)$ \\
\hline 6 & Total NPEV episodes & 162 & 115 & 76 & 50 & $403 / 4545(8.9)$ \\
\hline \multirow[t]{7}{*}{7} & NPEV+ve-acute episodes (\%) & 103/162 (63.6) & 83/115 (72.2) & 49/76 (64.5) & $33 / 50(66.0)$ & $268 / 403(66.5)$ \\
\hline & Acute diarrhoeal episodes & 19 & 13 & 10 & 6 & 48/403 (11.9) \\
\hline & NPEV-AIFoBM-ND/total & $25 / 61(41.0)$ & 21/61 (34.4) & 9/61 (14.8) & $6 / 61(9.8)$ & $61 / 115(53.0)$ \\
\hline & NPEV-IFoBM-ND episodes (\%) & & & & & \\
\hline & $\begin{array}{l}\text { NPEV-AIFoBM-ND/total NPEV } \\
\text { episodes (\%) }\end{array}$ & 25/162 (15.4) & $21 / 115(18.3)$ & 9/76 (11.8) & $6 / 50(12.0)$ & $61 / 403(15.1)$ \\
\hline & $\begin{array}{l}\text { NPEV-acute symptomatic } \\
\text { (IFoBM+diarrhoea) }\end{array}$ & 44 & 34 & 19 & 12 & $109 / 403(27.1)$ \\
\hline & $\begin{array}{l}\text { Acute asymptomatic NPEV } \\
\text { episodes }\end{array}$ & 59 & 49 & 30 & 21 & 159/403 (39.5) \\
\hline \multirow[t]{6}{*}{8} & $\begin{array}{l}\text { NPEV-positive persistent } \\
\text { episodes }\end{array}$ & 59 & 32 & 27 & 17 & 135 \\
\hline & NPEV-positive PD episodes (\%) & 7/162 (4.3) & $7 / 115(6.1)$ & $9 / 76(11.8)$ & 2/50) (4.0) & $25 / 403(6.2)$ \\
\hline & $\begin{array}{l}\text { NPEV-PIFoBM-ND/total } \\
\text { NPEV-PIFoBM-ND (\%) }\end{array}$ & $26^{*} / 54(48.2)$ & $12 / 54(22.2)$ & $9 / 54(16.7)$ & $7 / 54(13.0)$ & $54 / 115(47.0)$ \\
\hline & $\begin{array}{l}\text { NPEV-PIFoBM-ND/total NPEV } \\
\text { episodes (\%) }\end{array}$ & 26/162 (16.0) & $12 / 115(10.4)$ & 9/76 (11.8) & $7 / 50(14.0)$ & $54 / 403(13.4)$ \\
\hline & $\begin{array}{l}\text { NPEV-persistent symptomatic } \\
\text { (PD+PIFoBM-ND) }\end{array}$ & 33 & 19 & 18 & 9 & $79 / 403(19.6)$ \\
\hline & $\begin{array}{l}\text { Persistent asymptomatic NPEV } \\
\text { episodes }\end{array}$ & 26 & 13 & 9 & 8 & $56 / 403(13.9)$ \\
\hline 9 & Non-NPEV-IFoBM-ND episodes & 15 & 6 & 2 & 1 & 24/139 (17.3) \\
\hline \multirow[t]{3}{*}{10} & $\begin{array}{l}\text { NPEV-IFoBM-ND/total } \\
\text { NPEV-IFoBM-ND (\%) }\end{array}$ & $51 / 115(44.4)$ & 33/115 (28.7) & $18 / 115(15.6)$ & $13 / 115(11.3)$ & $115 / 403(28.5)$ \\
\hline & $\begin{array}{l}\text { NPEV-IFoBM-ND/total NPEV- } \\
\text { +non-NPEV-IFoBM-ND (\%) }\end{array}$ & $51 / 139(36.7)$ & 33/139 (23.7) & 18/139 (12.9) & $13 / 139(9.4)$ & $115 / 139(82.7)$ \\
\hline & $\begin{array}{l}\text { Total NPEV-IFoBM-ND/total } \\
\text { non-diarrhoeal episodes (\%) }\end{array}$ & $51 / 330(15.5)$ & $33 / 330(10.0)$ & $18 / 330(5.5)$ & $13 / 330(3.9)$ & $115 / 330(34.9)$ \\
\hline 11 & $\begin{array}{l}\text { NPEV diarrhoeal episodes/ } \\
\text { NPEV infection episodes (\%) }\end{array}$ & 26/162 (16.1) & $20 / 115$ (17.4) & $19 / 76(25.0)$ & $8 / 50$ (16.0) & 73/403 (18.0) \\
\hline \multirow[t]{2}{*}{12} & $\begin{array}{l}\text { NPEV symptomatic episodes/ } \\
\text { period }(\%)\end{array}$ & $77 / 162$ (47.5) & $53 / 115(46.1)$ & $37 / 76(48.7)$ & $21 / 50(42.0)$ & $188 / 403(46.7)$ \\
\hline & $\begin{array}{l}\text { NPEV symptomatic episodes/ } \\
\text { total NPEV episodes (\%) }\end{array}$ & $77 / 403$ (17.0) & $53 / 403$ (13.2) & $37 / 403(9.2)$ & $21 / 403(5.2)$ & \\
\hline \multirow[t]{3}{*}{13} & $\begin{array}{l}\text { NPEV asymptomatic episodes/ } \\
\text { period (\%) }\end{array}$ & $85 / 162(52.5)$ & $62 / 115(53.9)$ & $39 / 76(51.3)$ & $29 / 50(58.0)$ & 215/403 (53.3) \\
\hline & $\begin{array}{l}\text { NPEV asymptomatic episodes/ } \\
\text { total NPEV episodes (\%) }\end{array}$ & 85/403 (21.1) & $62 / 403(15.4)$ & $39 / 403(9.7)$ & $29 / 40(6.9)$ & \\
\hline & $\begin{array}{l}\text { NPEV asymptomatic episodes/ } \\
\text { non-diarrhoeal episodes (\%) }\end{array}$ & 85/330 (25.8) & $62 / 330(18.8)$ & 39/330 (11.8) & 29/330 (8.8) & $215 / 330(65.2)$ \\
\hline 14 & RV diarrhoea/RV episodes (\%) & $16 / 49(32.7)$ & $14 / 23(60.9)$ & $7 / 15(46.7)$ & $5 / 9(55.6)$ & 42/96 (43.7) \\
\hline 15 & NPEV-RV-positive episodes & 15 & 4 & 4 & 2 & $25 / 4545(0.5)$ \\
\hline 16 & $\begin{array}{l}\text { NPEV+RV-diarrhoeal episodes/ } \\
\text { total diarrhoeal episodes (\%) }\end{array}$ & 9/193 (4.6) & $1(0.5)$ & $1(0.5)$ & $1(0.5)$ & $12 / 193(6.2)$ \\
\hline 17 & $\begin{array}{l}\text { Diarrhoea due to other agents/ } \\
\text { total diarrhoea (\%) }\end{array}$ & 25/193 (12.9) & 20/193 (10.3) & $13 / 193(6.7)$ & 8/193 (4.1) & 66/193 (34.2) \\
\hline
\end{tabular}




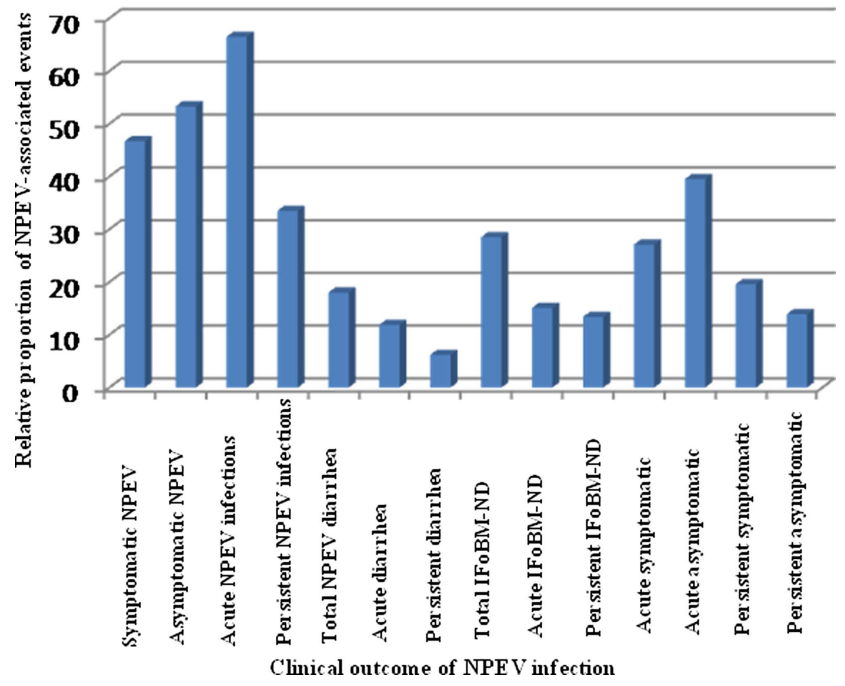

Figure 3 Per cent proportion of different non-polio enterovirus (NPEV)-associated symptoms with reference to total NPEV infection episodes in the cohort community during the 2-year follow-up period. Total number of samples, total NPEV-positive episodes, diarrhoeal episodes and oral polio vaccine samples in the follow-up study were described previously $^{30}$ (IFoBM, increased frequency of bowel movements).

monotype infections (table 2), $25 \%$ of PIFoBM-ND episodes were of monotype infections and $75 \%$ involved sequential infections by different strains. However, both types of infections occurred at a similar frequency in PD episodes. ${ }^{30}$ Among the agents examined, OPV strains, $\mathrm{RV}$ and diarrhoeagenic E. coli were detected in a small number of samples from a few episodes. When diarrhoeagenic E. coli was detected during some NPEV infection episodes, the stool was loose, but not diarrhoeal.

\section{DISCUSSION}

Among all bodily functions, BM is the most poorly studied and least understood. Although infectious and non-infectious causes may be associated with IFoBM, bacterial, parasitic and viral infections are frequently associated with diarrhoea (IFoBM-D). ${ }^{1}{ }^{2}{ }^{23-30} \quad 34-36$ To date, neither IFoBM-ND has been recognised as a clinical symptom nor a viral agent has been implicated to be associated with it. The present novel clinical finding of significant association of NPEV infection with IFoBM-ND in children strongly supports the personal experience and inconvenience suffered by DCR with the clinical symptom.

This study represents the first description of a possible viral aetiology of IFoBM-ND, both AIFoBM-ND and PIFoBM-ND, in infants and children. NPEV-associated IFoBM-ND episodes accounted for about $29 \%$ of NPEV infection episodes and $83 \%$ of total IFoBM-ND episodes (table 1). In about $17 \%$ of IFoBM-ND episodes, no NPEV or other examined agents were detected, suggesting the association of non-infectious causes or unknown agents with these episodes.

Of interest, 26 infants, included in the total number of NPEV-associated IFoBM-ND episodes, with a BM frequency of one in 4-10 days, had one or two normal stools per day following NPEV infection. However, no change in BM frequency was observed in nine children with constipation on NPEV infection. Thus, while about $74 \%$ of constipation episodes ${ }^{34}$ appear to have been resolved following NPEV infection, no effect was observed in $26 \%$ of constipation episodes on NPEV infection. To date, the clinical basis of constipation and their subsequent resolution in infants is poorly understood. The lack of change in BM frequency in these episodes could be due to the genetic diversity of the infecting NPEV isolates.

Recently, we reported a significant association of NPEV infections with acute diarrhoea as well as PD, and that every child in this cohort had at least one NPEV infection, symptomatic (diarrhoeal) and/or asymptomatic, by 2 years of age. ${ }^{23}$ Analysis of NPEV strains associated with IFoBM-ND episodes in the present study revealed the patterns of NPEV association with AIFoBM-ND and PIFoBM-ND episodes to be similar to that observed for PD episodes. While monotype infections were associated with about $25 \%$ of PIFoBM-ND episodes, sequential infections by different EV serotype strains were associated with $75 \%$ of episodes. By contrast, infection involving monotype strains and multiple strains occurred at a similar frequency in PD episodes. ${ }^{30} \mathrm{OPV}$ strains, RV and/or diarrhoeagenic E. coli were detected only in a small number of sequential samples from a few PIFoBM-ND episodes (table 2). It may be noted that two PIFoBM-ND episodes started as acute diarrhoea involving EV11 infection, but transformed into PIFoBM-ND after 4 days.

Although no child in the cohort developed serious complications during NPEV infection episodes, 20 children had three to four episodes of common cold, 23 had minimal non-febrile illness and 19 had respiratory illness, $80 \%$ of which coincided with NPEV infections. These events associated equally with diarrhoeal and nondiarrhoeal NPEV episodes. Twenty-seven babies had non-specific vomiting during the first 3 months without correlation to $\mathrm{EV}$ infection. No hand-foot-and-mouth disease or meningitis symptoms were observed in the cohort during this period. All the children were normal and healthy and did not suffer from inflammatory bowel disease, IBS or coeliac disease.

A notable finding was that NPEV is the single most frequently and persistently detected infectious agent in PIFoBM-ND episodes than any other agent we have examined in this study, similar to that observed in PD episodes. ${ }^{30}$ In this context, EVs are known to cause persistent infections in humans, animal models and cells in vitro with some strains capable of persisting for several months. ${ }^{39-47}$ Although it is considered that $<1 \%$ of $\mathrm{EV}$ infections result in severe disease with the majority 
Table 2 NPEV serotypes associated with acute diarrhoea, PD, AIFoBM, PIFoBM and asymptomatic infections, and the sequence of infections in PIFoBM episodes involving EV, RV and DEC

\begin{tabular}{|c|c|c|c|c|c|c|}
\hline EV-serotypes & $\begin{array}{l}\text { Strains in } \\
\text { acute \& PD } \\
\text { episodes } \\
\text { analysed }\end{array}$ & $\begin{array}{l}\text { Frequency of } \\
\text { detection of different } \\
\text { NPEV serotypes in } 48 \\
\text { independent } \\
\text { PIFoBM-ND episodes } \\
\text { analysed }\end{array}$ & $\begin{array}{l}\text { AIFoBM-ND } \\
\text { episodes } \\
\text { analysed }\end{array}$ & $\begin{array}{l}\text { Total } \\
\text { IFoBM-ND } \\
\text { samples/ } \\
\text { episodes } \\
\text { analysed }\end{array}$ & $\begin{array}{l}\text { Asymptomatic } \\
\text { EV episodes } \\
\text { analysed }\end{array}$ & $\begin{array}{l}\text { Total EV } \\
\text { samples/ } \\
\text { episodes } \\
\text { examined }\end{array}$ \\
\hline E1 & 2 & $\begin{array}{l}10 \text { (EV83, E1), (E1, } \\
33),(E 1,11),(E 32, \\
1,2), \\
(E 7,33,1),(E 1,2), \\
(E 1,25),(E 11,1), \\
(E 25,25,1,1,19, \\
E P E C, R V),(E 2,1),\end{array}$ & 10 & 20 & 10 & 32 \\
\hline E2 & 2 & $\begin{array}{l}3(E 2,1),(E 32,1,2) \\
(E 1,2)\end{array}$ & - & 3 & - & 5 \\
\hline E3 & $2+2$ & - & 1 & 1 & 1 & 6 \\
\hline E5 & 1 & - & - & - & - & 1 \\
\hline E6 & $5+10$ & $\begin{array}{l}13(E 6,6,6),(E 6,6,6), \\
(E 6,6, E P E C, R V), \\
(E 6,6,6),(E 6,6,6,6), \\
(E 21,6,6, O P V 1),(E 6, \\
19,19),(E 6,19),(E 6, \\
19, \text { OPV1), (E11, 11, } \\
6),(E 21,21,6),(E 11, \\
11, \text { OPV1, 6, EAEC), } \\
(E 19,19,19,19, R V, \\
\text { E6, ETEC) }\end{array}$ & 3 & 16 & 2 & 33 \\
\hline E7 & $2+8$ & $2(E 7,33,1),(E 21,7)$ & 2 & 4 & 1 & 15 \\
\hline E11 & $6+3$ & $\begin{array}{l}16(E 11,11),(E 11,11), \\
(E 11,11,11,11), \\
(E 11,1),(E 11,11,24), \\
(E 1,11),(E 25,11), \\
(E 11, C V A 9),(E 32,11), \\
(E 11,11,6),(E 11,11, \\
\text { OPV1, 6, EAEC), (E11, } \\
11,24,24),(E 11 D, 32, \\
\text { OPV1), (OPV1, E11, } \\
11),(E 11 D, 32, \text { OPV1), } \\
\text { (E11, RV) }\end{array}$ & 6 & 22 & 8 & 39 \\
\hline E12 & - & - & - & - & 2 & 2 \\
\hline E13 & $4+2$ & $1(\mathrm{E} 13,13)$ & 1 & 2 & 1 & 9 \\
\hline E14 & $2+1$ & $\begin{array}{l}4(E 14,14),(E 14,24), \\
(E 1,11,14),(E 14,19),\end{array}$ & 2 & 6 & 2 & 11 \\
\hline E19 & $3+3$ & $\begin{array}{l}6(E 6,19,19),(E 6,19), \\
(E 14,19)(E 25,25,1, \\
19,19, E P E C, R V), \\
(E 6,19, R V, O P V 1), \\
(E 19,19,19,19, R V, \\
\text { E6, ETEC) }\end{array}$ & 3 & 9 & 5 & 20 \\
\hline E20 & 2 & $2(E 20,20),(E 20,33)$ & 2 & 4 & 3 & 9 \\
\hline E21 & - & $\begin{array}{l}3(E 21,21),(E 21,6,6, \\
O P V 1),(E 21,21,6)\end{array}$ & 1 & 4 & 2 & 6 \\
\hline E24 & $3+3$ & $\begin{array}{l}6(E 24,24),(E 24,32), \\
(E 24,32),(E 11,11, \\
24),(E 14,24),(E 11, \\
11,24,24)\end{array}$ & 2 & 8 & 1 & 15 \\
\hline E25 & - & & 2 & 5 & 2 & 7 \\
\hline
\end{tabular}


Table 2 Continued

\begin{tabular}{|c|c|c|c|c|c|c|}
\hline EV-serotypes & $\begin{array}{l}\text { Strains in } \\
\text { acute \& PD } \\
\text { episodes } \\
\text { analysed }\end{array}$ & $\begin{array}{l}\text { Frequency of } \\
\text { detection of different } \\
\text { NPEV serotypes in } 48 \\
\text { independent } \\
\text { PIFoBM-ND episodes } \\
\text { analysed }\end{array}$ & $\begin{array}{l}\text { AIFoBM-ND } \\
\text { episodes } \\
\text { analysed }\end{array}$ & $\begin{array}{l}\text { Total } \\
\text { IFoBM-ND } \\
\text { samples/ } \\
\text { episodes } \\
\text { analysed }\end{array}$ & $\begin{array}{l}\text { Asymptomatic } \\
\text { EV episodes } \\
\text { analysed }\end{array}$ & $\begin{array}{l}\text { Total EV } \\
\text { samples/ } \\
\text { episodes } \\
\text { examined }\end{array}$ \\
\hline & & $\begin{array}{l}3(E 25,25,1,1,19, \\
E P E C, R V),(E 1,25), \\
(E 25,11)\end{array}$ & & & & \\
\hline E30 & 1 & - & - & - & 2 & 3 \\
\hline E32 & $1+1$ & $\begin{array}{l}4(E 32,11),(E 24,32), \\
(E 32,1,2)(E 11,32, \\
\text { OPV1) }\end{array}$ & 2 & 6 & 2 & 10 \\
\hline E33 & $4+2$ & $\begin{array}{l}3(E 7,33,1),(E 1,33), \\
(E 20,33)\end{array}$ & 3 & 6 & 2 & 14 \\
\hline EV75 & $3+2$ & - & - & - & - & 5 \\
\hline EV76 & - & - & - & - & 1 & 1 \\
\hline EV83 & - & 1 (EV83, E1) & 1 & 2 & 1 & 3 \\
\hline CVA4 & $1+1$ & - & - & - & 1 & 3 \\
\hline CVA9 & $1+1$ & - & 1 & 1 & 1 & 4 \\
\hline CVA13 & 1 & - & - & - & 1 & 2 \\
\hline CVA24 & $1+1$ & - & 1 & 1 & - & 3 \\
\hline CVB3 & 2 & - & 1 & 1 & 1 & 4 \\
\hline CVB4 & $1+2$ & & 1 & 1 & 1 & 5 \\
\hline CVB5 & $1+1$ & - & - & - & - & 2 \\
\hline OPV1 & $1+2$ & $\begin{array}{l}4 \text { (OPV1, E11, 11), } \\
\text { (E21, 6, 6, OPV1), } \\
\text { (E11D, 32, OPV1), (E6, } \\
19, \text { OPV1) }\end{array}$ & - & 4 & - & 7 \\
\hline OPV2 & 1 & - & - & - & - & 1 \\
\hline OPV3 & 1 & - & - & - & - & 1 \\
\hline Total & $\begin{array}{l}54+45=99 \\
(80)\end{array}$ & $\begin{array}{l}81 \text { (12 mono+69 } \\
\text { sequential) (48) }\end{array}$ & 45 & $126(91)$ & 53 & $278(222)$ \\
\hline \multicolumn{7}{|c|}{$\begin{array}{l}\text { The numbers in each row in each column represent the number of episodes from which the VP1 gene from strains belonging to the particular } \\
\text { EV serotype was sequenced. Strains indicated in parenthesis in italic font refer to sequential infections by more than one NPEV serotype or } \\
\text { agent in that order and those in normal font refer to monotypic infections. Strains from all the IFoBM-ND and asymptomatic NPEV infection } \\
\text { episodes were not examined. Numbers in italic font in the bottom row include some sequentially collected samples during persistent episodes } \\
\text { indicating representation of some episodes more than once, and the numbers in parenthesis in normal font in the bottom row indicate actual } \\
\text { number of PIFoBM-ND episodes analysed. In the second column, numbers in normal and italic fonts represent samples from acute and } \\
\text { persistent diarrhoeal episodes, respectively. } \\
\text { AIFoBM, acute increased frequency of bowel movements; CVA, coxsackievirus A; CVB, coxsackievirus B; DEC, diarrhoeagenic Escherichia } \\
\text { coli; E, echovirus; EAEC, enteroaggregative E. coli; EPEC, enteropathogenic E. coli; ETEC, enterotoxigenic E. coli; EV, enterovirus; } \\
\text { IFoBM-ND, non-diarrhoeal IFoBM; NPEV, non-polio enterovirus; OPV, oral polio vaccine; PD, persistent diarrhoea; PIFoBM, persistent IFoBM; } \\
\text { RV, rotavirus. }\end{array}$} \\
\hline
\end{tabular}

being asymptomatic, ${ }^{19}$ our recent and present results reveal that about $50 \%$ of NPEV infections are associated with significant gastrointestinal symptoms involving diarrhoea and IFoBM-ND. Although the precise molecular basis for the symptomatic/asymptomatic nature of the isolates is not understood, mutations in the virus ${ }^{44-52}$ and host factors could influence the replication and clinical outcome of the infection. ${ }^{47-52}$ Our recent studies showed that Indian isolates exhibit a significant genetic diversity from strains isolated in other countries, forming distinct genogroups or subgenogroups within a genotype. $^{22} 23$ This high level of genetic variation among isolates within a serotype could result in different isolates exhibiting a wide range of virulence phenotypes.
In conclusion, this study revealed (1) an unrecognised but highly significant clinical symptom, IFoBM-ND, in children with possible EV aetiology, (2) a similarity in BM frequency between diarrhoeal and IFoBM-ND episodes in children, the two symptoms differing in the type of stools passed, with the former characterised by watery stools and the latter by apparently normal stools, (3) NPEV as the single most frequently and persistently detected infectious agent in PIFoBM-ND and (4) an association of approximately $50 \%$ of NPEV infection episodes with gastrointestinal diseases involving diarrhoea and IFoBM-ND. PD and IFoBM-ND episodes could result in high morbidity and/or mortality in rural community settings, imposing extreme trauma and economic burden 
on poor families in developing countries. Our recent and present findings of significant NPEV association with acute and persistent symptomatic infections involving diarrhoea and IFoBM-ND in children are of great clinical significance and child health concern, warranting urgent global attention for detailed future investigations on these ubiquitous viruses which remained neglected and unrecognised as enteric pathogens.

Acknowledgements The authors are greatly indebted to the mothers of the cohort children for their interest, cooperation and support for this long-term study, without which this study could not have been possible. They thank A Raghavendra, Sudheendra Kumar, Harikrishna Reddy, E Surekha, G Ramya, $\mathrm{R}$ Bharat and V Vembuli for their technical support in processing of stool samples, for detection of NPEVs in cell culture primarily by DCR, analysis of stool samples for rotavirus by RNA PAGE and maintenance of sample records, and hence they were accorded coauthorship for their help in a previous manuscript. The authors are thankful to Mr Sanjay Kumar for collection and transport of stool samples from the residences of children according to schedule, and maintaining the details of information on OPV administration and samples at the hospitals. They also thank Dr A Srinivasa Rao, Georgia Regents University for help in statistical analysis of the data.

Contributors This project is an outcome of the personal clinical experiences of DCR, who is instrumental in designing, writing and securing funds for the implementation of the project's goals. DCR carried out an evaluation of a majority of the stool samples in cell culture, RNA extractions and RT-PCR, VP1 sequence and phylogenetic analyses, interpretation of data and writing of the manuscript. AMB and PPM were responsible for development and implementation of clinical protocols, selection, periodic monitoring and treatment of children during sickness and maintenance of clinical history of children, details of stool samples, and dates of OPV administration, and reviewed the manuscript.

Funding The work was supported by a grant (BT/01/COE/07/03) from the Department of Biotechnology, Government of India, from 2007 to 2012.

Competing interests None.

Patient consent Parental/guardian consent obtained.

Ethics approval Institutional Ethics committee, Indian Institute of Science.

Provenance and peer review Not commissioned, externally peer reviewed.

Data sharing statement Any data pertaining to OPV schedules, stool samples, NPEV strains and the cohort will be provided on request.

Open Access This is an Open Access article distributed in accordance with the Creative Commons Attribution Non Commercial (CC BY-NC 4.0) license, which permits others to distribute, remix, adapt, build upon this work noncommercially, and license their derivative works on different terms, provided the original work is properly cited and the use is non-commercial. See: http:// creativecommons.org/licenses/by-nc/4.0/

\section{REFERENCES}

1. Noseworthy J. Frequent bowel movements. 2014. http://www mayoclinic.com/health/frequent-bowel-movements/MY00786

2. Better Medicine from Health Grades Inc. Frequent bowel movementscauses_better medicine. http://www.localhealth.com/frequent-Bowel -movements/causes (accessed on 3 Mar 2014).

3. Heaton $\mathrm{KW}$, Radvan J, Cripps $\mathrm{H}$, et al. Defecation frequency and timing, stool form in the general population: a prospective study. Gut 1992;33:818-24.

4. Myo-Khin, Nyunt-T-W, Kyaw-Hla S, et al. A prospective study on defecation frequency, stool weight, and consistency. Arch Dis Child 1994;71:311-13.

5. Osatakul S, Yossuk P, Mo-Suwan L. Bowel habits of normal Thai children. J Pediatr Gastroenterol Nutr 1995;20:339-42.

6. Dukas I, Willett WC, Colditz GA, et al. Prospective study of bowel movement, laxative use, and risk of colorectal cancer among women. Am J Epidemiol 2000;151;958-64.
7. Camilleri $\mathrm{M}$, Lee JS, Viramontes $\mathrm{B}$, et al. Insights into the pathophysiology and mechanisms of constipation, irritable bowel syndrome, and diverticulosis in older people. J Am Geriatr Soc 2000;48:1142-50.

8. Dukas I, Leitzmann MF, Willett WC, et al. Association of bowel movement frequency and use of laxatives with occurrence of symptomatic gallstone disease in a prospective study of women. Am J Gastroenterol 2001;96:715-21.

9. Kitajima H, Ida S, Fujimura M. Daily bowel movements and Escherichia coli O157 infection. Arch Dis Child 2002;87:335-6.

10. Kojima M, Wakai K, Tokudome S, et al. Bowel movement frequency and risk of colorectal cancer in a large cohort study of Japanese men and women. Br J Cancer 2004;90:1397-401.

11. Lonstreth GF. Bowel patterns and anxiety. Demographic factors. J Clin Gastroenterol 1993;17:128-32.

12. Walker AR, Walker BF. Bowel behavior in young black and white children. Arch Dis Child 1985;60:967-70.

13. Fontana $\mathrm{M}$, Bianchi $\mathrm{C}$, Cataldo $\mathrm{F}$, et al. Bowel frequency in healthy children. Acta Paediatr Scand 1989;78:682-4

14. Tham EB, Nathan R, Davidson GP, et al. Bowel habits of healthy Australian children aged 0-2 years. J Paediatr Child Health 1996;32:504-7.

15. Benjasuwantep B, Ruangdaraganon N. Bowel movements in normal Thai infants. Southeast Asian J Trop Med Pub Health 2009;40:530-7.

16. Corazziari E, Staiano A, Miele E, et al. Bowel frequency and defecatory patterns in children. A prospective nationwide survey. Clin Gastroenterol Hepatol 2005;3:1101-6.

17. Tunc VT, Camurdan AD, Ilhan MN, et al. Factors associated with defecation patterns in 0-24-month-old children. Eur J Paediatr 2008;167:1357-62.

18. Wald ER, Jagodzinski TD, Moyer SC, et al. Validation and clinical utility of a bowel habit questionnaire in school-age children. J Paediatr Gastroenterol Nutr 1995;20:339-42.

19. Pallansch M, Roos R. Enteroviruses: polioviruses, coxsackieviruses, echoviruses, and newer enteroviruses. In: Knipe DM, Howley PM, Griffin DE, Martin MA, Lamb RA, Roizman B, Straus SE, eds. Fields virology. 5th edn. Philadelphia: Wolters Kluver, Lippincott, Williams \& Wilkins, 2007:839-93.

20. Adams MJ, King AMQ, Carstens EB. Ratification vote on taxonomic proposals to the International Committee on Taxonomy of Viruses. Arch Virol 2013;158:2023-30.

21. Chen TC, Shih SR. Drug discovery in enteroviral infections. Infect Disord Drug Targets 2011;11:337-45.

22. Rao CD, Yergolkar P, Shankarappa KS. Antigenic analysis of enteroviruses associated with nonpolio acute flaccid paralysis, India, 2007-2009. Emerg Infect Dis 2012;18:1833-40.

23. Rao CD, Ananda Babu M, Raghavendra A, et al. Non-polio enteroviruses and their association with acute diarrhea in children in India. Infect Genet Evol 2013;17:153-61.

24. Kumar A, Shukla D, Kumar R, et al. Molecular identification of enteroviruses associated with aseptic meningitis in children from India. Arch Virol 2013;158:211-15.

25. Kumar A, Shukla D, Kumar R, et al. Molecular epidemiological study of enteroviruses associated with encephalitis in children from India $J$ Clin Microbiol 2012:50:3509-12.

26. Patel JR, Daniel MJ, Mathan VI. An epidemic of acute diarrhea in rural southern India associated with echovirus type 11 infection. J Hyg (Lond) 1985;95:483-92.

27. Phan TG, Nguyen TA, Shimizu H, et al. Identification of enteroviral infection among infants and children admitted to hospital with acute gastroenteritis in Ho Chi Minh City, Vietnam. J Med Virol 2005;77:257-64.

28. Rai BS, Wan Mansor $\mathrm{H}$, Vasantha $\mathrm{T}$, et al. An outbreak of echovirus 11 amongst neonates in a confinement home in Penang, Malaysia. Med J Malaysia 2007;62:223-6.

29. Scarcella C, Carsi S, Cadoria F, et al. An outbreak of viral gastroenteritis linked to municipal water supply, Lombardy, Italy, June 2009. Euro Surveill 2009;14,Pii:19274.

30. Rao CD, Maiya PP, Ananda Babu M. Non-polio enterovirus association with persistent diarrhea in children as revealed by a follow-up study of an Indian cohort from birth to two years of life. J Clin Virol 2014;61:125-31.

31. Talley NJ. Irritable bowel syndrome. Intern Med J 2006;36:724-8.

32. Whitehead WE, Palsson O, Jones KR. Systematic review of the comorbidity of irritable bowel syndrome with other disorders: what are the causes and implications? Gastroenterol 2002;122:1140-56.

33. Holten KB, Wetherington A, Bankston L. Diagnosing the patient with abdominal pain and altered bowel habits: is it irritable bowel syndrome? Am Fam Physician 2003:67:2157-62.

34. Ambarlsumyan L, Rodriguez L. Gastrointestinal motility disorders in children. Gastroenterol Hepatol 2014;10:16-26. 
35. Mathai J, Raju B, Bavdekar A, Paediatric Gastroenterology Chapter Indian Academy of Pediatrics. Chronic and persistent diarrhea in infants and young children: status statement. Indian Pediatr 2011;48:37-42.

36. Fagundes-Neto U. Persistent diarrhea: still a serious public health problem in developing countries. Curr Gastroenterol Rep 2013;15:345

37. Tamura K, Peterson D, Peterson N, et al. MEGA5: molecular evolutionary genetics analysis using maximum likelihood, evolutionary distance, and maximum parsimony methods. Mol Biol Evol 2011;28:2731-9. Publication PDF at http://www.kumarlab.net/ publications

38. Good PI. Introduction to statistics through resampling methods and Microsoft Office Excel. Hoboken, New Jersey: John Wiley \& Sons, Inc, 2005.

39. Martin J. Vaccine-derived poliovirus from long-term excretors and the end game of polio eradication. Biologicals 2006;34:117-22.

40. Zhang S, Racaniello VR. Persistent echovirus infection of mouse cells expressing the viral receptor VLA-2. Virology 1997;235:293-301.

41. Harrath R, Douche-Aourik F, Bourlet T, et al. Model of coxsackievirus B3 persistent infection in orally-inoculated BALB/C mouse. Ann Biol Clin 2004;62:33-9.

42. Li J, Lin C, Qu M, et al. Excretion of enterovirus 71 in persons infected with hand, foot and mouth disease. Virol J 2013;10:31.

43. Pavio N, Couderc T, Girard S, et al. Expression of mutated poliovirus receptors in human neuroblastoma cells persistently infected with poliovirus. Virology 2000;274:331-42.
44. Borzakian S, Pelletier I, Calvez V, et al. Precise missense and silent point mutations are fixed in the genomes of poliovirus mutants from persistently infected cells. J Virol 1993;67:2914-17.

45. Duncan G, Pelletier I, Colbère-Garapin F. Two amino acid substitutions in the type 3 poliovirus capsid contribute to the establishment of persistent infection in HEp-2c cells by modifying virus-receptor interactions. Virology 1998;241:14-29.

46. Pelletier I, Duncan G, Colbère-Garapin F. One amino acid change on the capsid surface of poliovirus sabin 1 allows the establishment of persistent infections in HEp-2c cell cultures. Virology 1998;241:1-13.

47. Schmidtke M, Merkle I, Klingel K, et al. The viral genetic background determines the outcome of coxsackievirus B3 infection in outbred NMRI mice. J Med Virol 2007;79:1334-42.

48. Ramsingh Al, Collins DN. A point mutation in the VP4 coding sequence of coxsackievirus B4 influences virulence. J Virol 1995;69:7278-81.

49. Coleman JR, Papamichail D, Skiena S, et al. Virus attenuation by genome-scale changes in codon pair bias. Science 2008;320:1784-7.

50. Song Y, Liu Y, Ward CB, et al. Identification of two functionally redundant RNA elements in the coding sequence of poliovirus using computer-generated design. Proc Natl Acad Sci USA 2012;109:14301-7.

51. Munnich LL, Ray CG. Variable susceptibility of mice to group B coxsackievirus infections. J Clin Microbiol 1980;11:73-5.

52. Yetterberg SR, Mahowald ML, Messner RP. Coxsackievirus B1-induced polymyositis: lack of disease expression in nu/nu mice. $J$ Clin Invest 1987;80:499-506. 